\title{
Variations
}

Variations

Revue internationale de théorie critique

12 | 2008

Wärmestrom

\section{Ce vent qui nous fouette}

La question des révoltes urbaines

\section{Jock Young}

Traducteur : José Chatroussat

\section{OpenEdition}

\section{Journals}

Édition électronique

URL : http://journals.openedition.org/variations/240

DOI : 10.4000/variations. 240

ISSN : 1968-3960

\section{Éditeur}

Les amis de Variations

\section{Édition imprimée}

Date de publication : 21 décembre 2008

\section{Référence électronique}

Jock Young, "Ce vent qui nous fouette », Variations [En ligne], 12 | 2008, mis en ligne le 01 janvier 2012, consulté le 20 avril 2019. URL : http://journals.openedition.org/variations/240 ; DOI : 10.4000/ variations. 240

Ce document a été généré automatiquement le 20 avril 2019.

Les ami•e•s de Variations 


\title{
Ce vent qui nous fouette
}

\author{
La question des révoltes urbaines
}

Jock Young

Traduction : José Chatroussat

\section{NOTE DE L'ÉDITEUR}

Traduit de l'anglais

Première publication sur www.theoriecritique.com, «Wärmestrom, le courant chaud en sciences humaines ", Hiver 2008/2009, pp. 47-57

"Crossing the borders : To these wet and windy shores » du livre de Jock Young, The vertigo of late modernity, SAGE, New York City, 2006. Nous remercions l'auteur et les éditeurs, en particulier Caroline Porter, d'avoir autorisé la publication de la présente version française inédite.

\section{Il y a plus de 20 ans : les émeutes de 1981}

1 Tout d'abord permettez-moi de revenir à l'analyse des émeutes de 1981 dont les principaux lieux se situaient à Brixton, Moss Side et Toxteth (voir Lea et Young, 1982). Les émeutiers étaient de façon prédominante des Afro-Caribéens avec une minorité blanche significative. Les émeutes furent dirigées contre la police et s'accompagnèrent de pillages de magasins. Une explication seulement en termes de pauvreté était insuffisante, dans la mesure où historiquement il $\mathrm{y}$ a eu pauvreté et chômage pires dans un Royaume-Uni sans émeutes. Cela n'explique pas non plus pourquoi au début des années 80 , certains secteurs de la population, en particulier des Afro-Caribéens, et non d'autres tels que les Asiatiques, participent aux émeutes. Cela n'explique pas non plus l'absence d'émeutes dans des zones telles que celle de Glasgow où prédomine une population pauvre et blanche.

2 La thèse conservatrice - la plus fortement soutenue à l'époque par la correspondante du Daily Telegraph, Peregrine Worsthorne - était qu'un échec s'était produit dans les relations 
entre races à cause d'un manque de politiques d'assimilation. À cause de politiques pluralistes, multiculturelles, les gouvernements successifs avaient permis aux cultures immigrées de maintenir ce qui était essentiellement «étranger » et ne s'identifiant pas à la culture britannique. Ainsi, lorsque s'exerça la pression de la pauvreté et du chômage, ce pluralisme relâché s'est simplement écroulé. On pointait là le contraste avec les EtatsUnis des années 50 où les politiques d'assimilation avaient été vigoureusement poursuivies: le drapeau était déployé dans les classes et les bâtiments publics, les récitations de la constitution et la célébration de la citoyenneté étaient de rigueur.

Notre critique de tout cela était que l'explication des événements ne résidait pas dans un malaise à cause d'un manque d'assimilation mais plutôt qu'il y avait un malaise à cause du degré d'assimilation. C'est la raison pour laquelle le malaise était le plus fort parmi les immigrés de la seconde génération qui avaient intégré les notions de citoyenneté dans tous ses aspects économiques, sociaux et politiques. C'était une émeute de la citoyenneté contrariée, et non pas une émeute due à un échec dans la compréhension du sens de la citoyenneté. Elle impliquait une génération d'Afro-Caribéens qui avaient fait l'expérience du manque de travail alors qu'ils s'attendaient à avoir un emploi, l'expérience du racisme alors qu'ils croyaient en l'égalité et l'expérience du harcèlement par la police alors qu'ils s'attendaient au respect du rôle de la loi. Replaçons à présent tout cela dans une perspective plus générale.

\section{Criminalité, immigration et diabolisation de l'autre}

Dans tout le monde occidental le processus de globalisation économique a été associé à la migration en masse de travailleurs du Tiers Monde et des pays du Deuxième Monde : les nations ex-soviétiques de l'Europe de l'Est. Dans tous les cas un processus social et spatial d'exclusion s'est produit dans le pays d'«accueil» et, lié à cela, le rejet culturel de l'«autre ", de la population immigrée. Il s'agit de la désignation de l'immigré comme étant un Autre, constitutif d'un groupe étranger et extérieur opposé à une soi-disant normalité culturelle de la population autochtone. Une série d'oppositions binaires s'établissent : eux-nous, majorité-minorité, pur-impur, et de façon soi-disant inévitable, conforme à la loi-criminel, normal-déviant. La population immigrée est perçue comme une source de criminalité, de drogues, de prostitution et de violence. Mettons les choses au clair: la recherche en Grande-Bretagne indique sans aucune équivoque que la première génération des immigrés a un taux de criminalité inférieur à celui de la population autochtone. En effet, comme Dario Melossi (2000) l'a indiqué, une telle conclusion s'applique à l'histoire de l'immigration aux USA. Elle a été relevée à la fois par l'Immigration Commission de 1911 et la Wickersam Commission de 1931, et en GrandeBretagne elle fut une conclusion cruciale en 1972 du Select Committee on Race Relations (voir Lea et Young, 1993, pp.135-8). Comme nous l'avons argumenté dans What is to be Done About Law and Order? (1993), c'est la seconde génération d'immigrés, qui a davantage assimilée les valeurs de la société dans son ensemble, qui ressent avec le plus d'acuité les privations; les mécontentements consécutifs conduisent fréquemment à des taux de criminalité plus élevés. Bien plus, cela n'est pas le produit d'on ne sait quelle essence raciale mais de sous-cultures qui se sont adaptées au nouveau pays et qui transforment plutôt qu'elles ne reproduisent la culture d'origine (ibid. pp. 124-9). De façon semblable Ineke Haen Marshall, dans son enquête sur les taux de criminalité au sein des minorités aux Etats-Unis et en Europe, relève : 
Il ressort un consensus général sur le fait que, s'il y a des différences marquées entre l'implication criminelle des immigrés et des autochtones, elles se manifestent dans l'implication criminelle des enfants d'immigrés (seconde - et troisième génération d'immigrés). Il est avéré que les enfants d'immigrés auront eu de plus fortes attentes; ils auront changé leurs aspirations dans l'existence. (1997, pp. 237-8 ; voir aussi Karydis, 1996)

Ajoutez à cela les tourments et difficultés liés à l'habitat aussi bien dans les centres des grandes villes que dans les zones périphériques et vous obtenez la formule d'un désastre.

En outre, les types de crimes qui se produisent de façon prépondérante sont liés démographiquement aux jeunes et aux difficultés liées à la pauvreté. Maintes et maintes fois, les déterminants de classe se mêlent aux tendances ethniques ou de « race » (voir Mooney, 2000).

\section{Les racines du rejet de l'autre}

7 Comment peut-on expliquer ce processus culturel de «rejet de l'autre »? Des tentatives sont souvent faites pour expliquer de telles tendances d'utiliser les théories psychanalytiques de Melanie Klein, Erik Erikson et plus récemment Julia Kristeva. Ces théories, dans la tradition de la théorie des relations objectives, tracent des parallèles entre le développement chez les enfants du sens de soi se distinguant du monde extérieur et les démarcations culturelles entre "nous » et «l'autre». Une explication alternative enracinée dans l'anthropologie culturelle et le travail de Mary Douglas situe de telles exclusions dans le besoin pour des groupes sociaux de maintenir des frontières et elle détaille comment les règles sur la pureté, la peur et la répugnance des autres renforcent la sécurité de la majorité retranchée.

8 Le problème avec une telle théorisation, défendue de la façon la plus éloquente dans le livre de David Sibley Geographies of Exclusion (1995), est qu'elle tend à décrire un tel rejet de l'autre ou diabolisation comme une donnée culturelle universelle, un produit des problèmes toujours présents de la psychologie humaine ou de la formation d'un groupe. $\mathrm{Au}$ lieu de cela je souhaite situer un tel processus dans le temps et dans un contexte social, pour spécifier qui est diabolisé avec davantage de probabilité, pour expliquer le contexte des qualificatifs appliqués aux étrangers, pour comprendre et décrire le résultat probable d'un tel rejet de l'autre. En bref, pour connaître quand, pourquoi, qui, comment et où s'effectue la diabolisation.

9 J'ai essayé de situer sociologiquement les racines du rejet de l'autre ${ }^{1}$. La désincarnation de la société post-moderne, le choc du pluralisme, la peur de la perte de statut toujours possible, ou de la mobilité vers le bas de l'échelle génèrent un vertige d'insécurité. Une solution à une telle perte d'identité ferme sont le nationalisme, le fondamentalisme, le racisme (et pour les hommes la virilité exacerbée), qui mènent à construire - ou à « inventer » comme l'ont voulu Hobsbawm et Ranger (1983) - une identité fixe fondée sur la notion d'essence culturelle qui est réaffirmée, redécouverte et sur laquelle on s'élabore. Cette essentialisation du soi, l'attribution à soi-même et à ses parents et amis de vertus solides enracinées dans sa culture, s'accompagnent inévitablement d'une essentialisation et d'un dénigrement de l'autre. Le processus de constitution du diable continue ainsi rapidement. La notion la plus simple de ce qui constitue un démon, un diable populaire, un ennemi dans chaque culture particulière est qu'il est ce qu'elles ne sont pas. C'est l'incarnation de tout ce contre quoi elles sont, une violation de leurs principes les plus 
élevés, leur morale et leurs valeurs - bref, il est constitué par la négativité - c'est le noir et blanc de la photographie morale.

Essayons d'aller un peu plus loin pour approfondir notre compréhension du rôle de l'immigré dans le processus de rejet de l'autre. Jusqu'à maintenant j'ai discuté de la genèse du rejet de l'autre mais il est important d'insister aussi sur comment les reproches à l'immigré s'ajoutent de façon très utile au discours insidieux centré sur les crimes et les désordres. Comme je l'ai argumenté ailleurs, si l'on part de la position que les sociétés du Premier Monde sont caractérisées par des inégalités considérables et par des stigmatisations largement répandues (injustice dans la répartition et la reconnaissance), alors l'existence de désordres n'est guère surprenante. C'est la position de la criminologie critique. Cependant, si l'on part d'une position plus conforme à l'ordre établi, à savoir que notre monde social, certes imparfait, est le meilleur des mondes possibles, alors le désordre, bien que compréhensible dans le Tiers Monde, devient problématique dans le Premier. Pour cette raison le discours sur la "guerre contre la criminalité » doit expliquer la déviance sans indiquer de remède. Il doit accomplir ce que Berger et Luckman (1966) appellent un exercice de "maintenance de l'univers symbolique ». La technique pour résoudre ce problème tient en deux volets. Tout d'abord la prise de distance : montrer du doigt les facteurs et les groupes qui sont repérés comme détachés du fonctionnement normal de l'ordre social. Un exemple de cela serait l'invocation presque magique du «gang » dans les médias américains et, de façon plutôt scandaleuse, dans la criminologie académique (pour une telle critique voir Kontos, Brotherton et Barrios, 2003). Deuxièmement l'inversion: à la place des problèmes de société engendrant la criminalité, la criminalité est considérée comme un problème autonome pour la société.

Un exemple clef de ces deux volets est la rhétorique sur la guerre contre les drogues. L'usage des drogues est considéré comme générant des crimes, ce qui crée des problèmes pour la société, c'est-à-dire :

Drogues $\rightarrow$ criminalité $\rightarrow$ problèmes sociaux

Plutôt que :

Problèmes de la société $\rightarrow$ drogues $\rightarrow$ criminalité

12 A présent, l'autre et/ou l'immigré, apportent une contribution très utile à ce genre de discours de la dénégation. En tant qu'étranger, élément extérieur, l'immigré (Albanais, Jamaïcain, etc.) apporte la criminalité dans la société, ce n'est pas de toute évidence un problème lié à l'ordre social mais le problème d'un groupe importé dans la société, c'està-dire :

Immigration $\rightarrow$ criminalité $\rightarrow$ problèmes sociaux

$\mathrm{Ou}$, mieux encore :

Immigrés $\rightarrow$ drogues $\rightarrow$ criminalité $\rightarrow$ problèmes sociaux

Dans ce processus de rejet de l'autre, plutôt que de faire face à l'ensemble du poids des problèmes sociaux de notre société (par exemple habitat dégradé, éducation inadapté, taux inférieur d'accès aux dispositifs de santé, taux élevés de criminalité et d'incivilités) l'immigré est alors envisagé comme étant la cause de ces problèmes.

\section{La phase finale : I'ironie de l'assimilation}

La grande ironie du rejet culturel de « l'autre » est qu'à mesure où les groupes d'immigrés s'assimilent davantage à la culture majoritaire, ils font l'expérience de niveaux plus élevés de pertes relatives et de malaise en réponse à leur pauvreté, et leur niveau de 
criminalité s'accroît. Ainsi la seconde génération des Afro-Caribéens en Grande-Bretagne a un niveau plus élevé de criminalité. De façon similaire, comme nous l'avons vu, les études de Carl Nightingale des Afro-Américains de Philadelphie (1993), et celles de Philippe Bourgois des Parto-Ricains à East Harlem (1995), montrent que le mécontentement social et la criminalité ne se produisent pas à cause d'une simple exclusion mais à l'inverse, à cause d'une intégration culturelle largement répandue faisant suite à une exclusion structurelle : ce que j'ai appelé la boulimie de la modernité tardive. Finalement, bien sûr, quelques groupes minoritaires s'emparent des caractéristiques négatives projetées sur eux, tandis que d'autres développent des cultures de la différence et prennent en charge des fondamentalismes pour donner un sens à leur rejet. Ayant convoqué et mise en avant de faux démons, nous nous retrouvons avec des démons réels se dressant en face de nous.

\section{Les racines des troubles}

L'interprétation des troubles parmi les groupes d'immigrés est, par conséquent, un rejet de «l'autre ». Incivilités, criminalité, émeutes sont vues comme un produit du manque d'assimilation des valeurs et des vertus civiques du pays d'accueil. C'est un produit de leur inconciliable "étrangeté». La réponse politique dans le cas des conservateurs implique l'accent mis sur l'assimilation par " par soi-même "; dans le cas du New Labour, avec son discours dominant sur l'inclusion sociale et sa confiance dans le multiculturalisme, l'accent est mis sur l'intégration et l'inclusion sociale. Pour les conservateurs l'assimilation est l'équivalent d'un exorcisme social, la chasse aux spécificités étrangères : la conversion du diable en un être civilisé. Pour les libéraux, l'inclusion s'opère par la correction du déficit. Dans ces deux exemples politiques, les racines des troubles sont «là-bas, en dehors ", enracinées dans des différences relevant de l'inadaptation. Ce que je défends est totalement à l'opposé de cela: les troubles arrivent à cause du degré d'assimilation; ils sont fonctions du fait de vouloir être davantage "comme nous " plutôt que d'être différents de nous. C'est l'assimilation ou l'intégration qui permet l'exclusion structurelle et le manque d'opportunités pour obtenir un travail ou le refus d'être acceptés comme citoyens qui conduit à faire l'expérience de l'injustice. Les jeunes Asiatiques qui ont participé aux émeutes dans les villes du nord avaient les mêmes attentes que les jeunes blancs qui y participaient de l'autre côté de la ligne ethnique. Ils n'avaient pas trop besoin d'apprendre la citoyenneté ou l'anglais - ils savaient très bien qu'une mauvaise police en action constituait une violation de leur citoyenneté au même titre que leur colossale exclusion des marchés du travail national.

Comment un tel processus d'assimilation des valeurs et aspirations occidentales s'est-il produit? Il s'est opéré sans qu'aucun gouvernement n'ait besoin d'intervenir, par l'exposition, particulièrement pour la seconde génération, aux mass media, à l'éducation de masse avec son curriculum national, au vaste appareil de la société de consommation avec ses publicités, ses centres commerçants et les images de réussites, de statuts visibles, matérialisés par des vêtements, des voitures et des accessoires de mode. Contre ce barrage culturel écrasant, global dans ses dimensions et intense par son impact, la possibilité pour n'importe quel groupe d'immigrés de rester séparé avec son identité est extrêmement limitée. Le paradoxe est alors le suivant. Tandis que les immigrés de la seconde génération deviennent de plus en plus proches des "hôtes" et que leurs aspirations économiques et politiques coïncident avec la société plus large, ils doivent 
faire face à la fois à une exclusion culturelle à cause du racisme et des préjugés et ils deviennent conscients du caractère limité pour eux des opportunités économiques. Cette insatisfaction au lieu de trouver une expression politique, une compréhension claire, de la situation difficile commune aux gens pauvres et socialement exclus quelle que soit leur origine ethnique (y compris la population autochtone), se transforme en une situation de ségrégation où des groupes socialement exclus entrent en conflit. Dans ce cas, les différences culturelles bien que restreintes sont grotesquement exagérées et les différences économiques bien que limitées sont perçues comme énormément injustes et disproportionnées.

\section{Les émeutes de Bradford, Oldham et Burnley}

17 Les émeutes qui se sont produites à Bradford, Oldham et Burnley en 2001 ont été d'une nature différente de celle des troubles qui se sont produits en Grande-Bretagne en 1981 et 1985. Les émeutes des années 80 étaient des émeutes d'inclusion, elles étaient une lutte contre le catalogue complet des exclusions sociales: racisme policier, chômage, marginalisation et impuissance politiques. Elles n'ont pas été propagées par le racisme mais contre le racisme. Par contraste avec elles, celles qui se sont produites plus récemment dans nos villes du nord ont un aspect plus sinistre; des secteurs de la communauté se sont dressés l'un contre l'autre. Des stéréotypes racistes et des préjugés ethniques ont été mobilisés et le but des deux côtés a alors été d'exclure, de séparer et de diviser. Ainsi, tandis que les soulèvements des années 80 ne furent pas et de loin des émeutes raciales, celles d'aujourd'hui sont à deux doigts d'appartenir à cette catégorie. De tels événements offrent des opportunités dangereuses pour les partis d'extrême droite; mais ils sont les bénéficiaires de tels conflits intercommunautaires plutôt qu'ils n'en sont les causes. Bien entendu le chômage et l'exclusion économique se trouvent derrière les événements des années 80 comme derrière ceux d'aujourd'hui. Mais la forme des troubles est très différente et c'est là que les problèmes d'identité et de multiculturalisme ont eu leur impact.

Loïc Wacquant dans son étude comparative célèbre entre Woodlawn dans le South Chicago et La Courneuve au-delà du périphérique de Paris, relève le contraste dramatique entre l'extrême ségrégation à Chicago et la population mélangée de Paris. "Les enclaves raciales, note-t-il, sont inconnues en France et dans toute l'Europe pour cette raison » (1996, p.560). La diversité des populations des grandes métropoles européennes est un des résultats les plus significatifs - bien qu'involontaire - de la modernité tardive. En effet je suis en train d'écrire cela à Stoke Newington, dans le quartier londonien de Hackney où, si vous prenez les questions de classe et d'ethnicité en considération, il n'y a probablement pas une population majoritaire - il s'agit d'une circonscription électorale à mixité diversifiée, une enclave de minorités. Mais les observations de Wacquant, bien qu'elles soient vraies pour de grands secteurs de nos grandes villes et renforcées par le taux élevé de mariages et d'amitiés mixtes, ne le sont pas pour certaines zones où les conditions de logement, les écoles et les peurs racistes ont commencé à créer une ségrégation et un mono-culturalisme. Bradford et oldham en sont des exemples, tandis que Belfast et Derry représentent les situations les plus exceptionnelles.

19 Aux Etats-Unis le degré exceptionnel de ségrégation spatiale a été sous-évalué par une idéologie du multiculturalisme et du communautarisme. Comme l'ont souligné des écrivains aussi divers que Zygmunt Bauman, Robert Hughes, Paul Gilroy et Tod Gitlin, les 
notions classiques de multiculturalisme, bien que libérales dans leur intention, ont potentiellement des conséquences réactionnaires. Dans un monde moderne tardif où les gens créent de plus en plus leur propre sens de l'identité et de la culture, le multiculturalisme encourage exactement l'opposé, pour rechercher vos racines et trouver votre " vrai » moi. Une telle essence immuable est alors en opposition avec « les autres » (Catholiques contre Protestants, Islam contre non-Islam, Blancs contre Noirs) et permet des préjugés fondés sur des notions de différences immuables. Un multiculturalisme qui aspire à la tolérance crée paradoxalement les conditions favorables aux préjugés et à l'intolérance.

20 Une solution à ce problème est le communautarisme, une mosaïque de communautés séparées, chacune homogène avec ses propres valeurs, et en sûreté à l'intérieur de leur propre identité. Mais comme l'expérience des USA l'a montré, la mosaïque se délite constamment sur les bords ; il y a, pour reprendre la phrase obsédante de Tod Gitlin, un «crépuscule des rêves communs » (1995), chaque communauté prenant ses dispositions contre les autres en adoptant des attitudes compétitives, exclusives et hostiles.

21 Que pouvons-nous faire à ce sujet? Tout d'abord nous avons besoin de résoudre le problème de l'exclusion économique qui nourrit les antagonismes entre les communautés. Comme nous l'avons vu, une inclusion économique doit impliquer de véritables possibilités d'améliorations éducatives, économiques et sociales. Elle ne peut pas simplement impliquer, au nom de la flexibilité du travail, le transfert de toute une catégorie de gens tout en bas sur le marché du travail. Deuxièmement, nous devons nous attaquer à la notion de fond des communautés multiculturelles qui dégrade tant la réflexion courante - à gauche et à droite - et qui facilite de tels antagonismes. La solution rationnelle à un monde divisé en entités binaires - eux et nous - comme la philosophe radicale new-yorkaise Nancy Fraser l'a souligné (1997), est de déconstruire les entités binaires - et non pas de les étayer. Cela implique de nous fixer pour objectif une nouvelle sorte de multiculturalisme - un multiculturalisme fondé sur une véritable diversité. Une société diverse est une société où il $\mathrm{y}$ a un véritable choix, où il $\mathrm{y}$ a un mélange de traditions, où l'accent est mis sur la création en cours de la culture plutôt que sur l'héritage d'une tradition pesante. Une société diverse, ce n'est pas Oldham ou Bradford où des cultures immuables et monolithiques s'affrontent. Ce n'est pas non plus le néotribalisme de l'Irlande du Nord où la tradition est glorifiée et où les problèmes d'identité sont soi-disant résolus en consultant les contours géographiques fixés sur un atlas. Par contraste, concernant une véritable diversité culturelle, il s'agit de créer de nouveaux styles de vie et de nouvelles valeurs, de bricoler des morceaux ici et là, il s'agit de l'hybridation de la culture plutôt que d'être en quête d'une fausse authenticité. C'est en fait l'actuelle culture vivante que les jeunes gens des écoles qui recrutent dans un environnement ethnique et de classe large, créent chaque jour de la semaine. L'ennemi de cette diversité, ce sont les politiques de logement ségrégatives, les écoles à confession unique, les leaders réactionnaires visant une communauté, et par-dessus tout, la fonction spécieuse de gens établissant des catégories ethniques.

Ce n'est pas la vieille histoire de l'assimilation, lorsque le pays «d'accueil » absorbe la minorité immigrée. Ce n'est pas ce que la journaliste du Daily Mail Melanie Phillips prescrit à propos des émeutes : une nouvelle assimilation - un melting-pot - où tout le monde devient culturellement «blanc» et terriblement Anglais. Avec la politique de la diversité, tout le monde change et la marque du progrès est un multiculturalisme qui 
chevauche, brouille et mélange, qui ne réinvente pas constamment le passé mais regarde en avant vers le futur.

Ce n'est pas le manque de connaissance de la citoyenneté qui a généré les émeutes dans les villes du nord mais exactement l'inverse : une forte notion apprise de l'économie, une citoyenneté politique et social contrariée. Ce qui est nécessaire est une notion d'inclusion sociale qui s'attaque à la fois à l'aliénation des communautés immigrées et au racisme de la population autochtone, l'éducation et les emplois nécessaires, l'isolement économique et géographique de telles villes et des politiques qui garantissent un encadrement policier et une intégration politique qui soient justes. De plus, le multiculturalisme classique qui se focalise sur une mosaïque de communautés séparées et statiques doit être remplacé par un multiculturalisme qui s'appuie sur les interactions, les changements, la diversité choisie. Tout cela, aussi bien en termes d'emplois, de police, de politique, d'opposition à la ségrégation sociale - dans l'espace public comme dans les écoles - exige que non seulement les simples citoyens prennent leurs responsabilités mais aussi que le gouvernement prenne ses responsabilités en fournissant des structures qui faciliteront les droits des citoyens et une véritable inclusion sociale.

\section{Post-scriptum : les émeutes en France en 2005}

Les émeutes en France qui ont commencé dans la banlieue de Paris et se sont propagées dans tout le pays, ont suscité de rudes réactions au sein de la République et d'étranges commentaires à l'extérieur. Le ministre de l'Intérieur, Nicolas Sarkozy, l'ex-aristocrate hongrois, candidat à la présidence en France, a parlé de "nettoyer la racaille au Karcher ». Le Karcher est un nettoyeur industriel que l'on pourrait utiliser pour enlever les déjections d'oiseaux sur un mur; la phrase a un relent de nettoyage ethnique. Les commentaires de l'autre côté de la Manche étaient largement à l'autocongratulation. Le Guardian dans son éditorial soutenait que la France devrait apprendre de la GrandeBretagne tout comme la Grande-Bretagne avait appris des USA. Il citait les émeutes de Detroit et Brixton et les enquêtes qui suivirent comme une évidence. Il ciblait la faillite du modèle de marché social et affirmait que : «La France n'a jamais eu à aucun moment l'approche multiculturelle de la Grande-Bretagne... Mais ce qui s'est passé en France renforce la justesse de nos objectifs actuels. » (9 novembre 2005).

Relevons tout d'abord que ce genre de comparaison confine à l'absurde. Detroit, la plus pauvre des grandes villes des Etats-Unis, avec un niveau de pauvreté pire qu'à la Nouvelle-Orléans, ne peut guère être montrée en exemple pour analyser le modèle social français de l'Etat providence. De plus, il est sidérant de mettre en avant le succès du multiculturalisme britannique étant donné le passé récent. Ainsi Jonathan Freedland (Le Guardian, 9 novembre) nous présente une analyse intéressante des émeutes françaises mais ses conclusions me déconcertent absolument. Pour avancer que le "multiculturalisme britannique » est "encore le meilleur modèle que nous ayons ", il faut ignorer le fait qu'il y a six ans comme il a été rappelé nous avons fait l'expérience d'émeutes violentes à Bradford, Oldham et Burney basées sur les divisions raciales, fortement exacerbées par le racisme et l'agressivité de la police. Une telle frustration a continué et a suppuré, ce qui a culminé dans les attentats à la bombe à Londres en juillet 2005 sur lesquels il a écrit de façon si vivante et perspicace. des contradictions de notre société : les idéaux d'intégration et la boulimie de rejet. Nous 
n'avons pas beaucoup appris des émeutes de Detroit, Brixton ou Bradford. Maintenant, nous devrions essayer d'en apprendre quelque chose.

NOTES

1. Jock Young, The Vertigo of Late Modernity, 2007, Search Publication Ltd.

\section{AUTEURS}

JOCK YOUNG

Professeur de sociologie, Londres 